TRANSACTIONS OF THE

AMERICAN MATHEMATICAL SOCIETY

Volume 352, Number 7, Pages 3103-3116

S 0002-9947(00)02589-7

Article electronically published on February 25, 2000

\title{
ADAMS OPERATIONS, LOCALIZED CHERN CHARACTERS, AND THE POSITIVITY OF DUTTA MULTIPLICITY IN CHARACTERISTIC 0
}

\author{
KAZUHIKO KURANO AND PAUL C. ROBERTS
}

\begin{abstract}
The positivity of the Dutta multiplicity of a perfect complex of $A$-modules of length equal to the dimension of $A$ and with homology of finite length is proven for homomorphic images of regular local rings containing a field of characteristic zero. The proof uses relations between localized Chern characters and Adams operations.
\end{abstract}

\section{INTRODUCTION}

Multiplicities defined by limits of Euler characteristics over powers of the Frobenius map in positive characteristic were introduced by Dutta [1, who showed that in many respects they have better properties than ordinary Euler characteristics. In particular, if $A$ is a local ring of dimension $d$, the Dutta multiplicity of a nonexact complex of free modules of length $d$ with homology of finite length is always positive; this fact was proven and used in an essential way to prove the Intersection Theorem for rings of mixed characteristic by Roberts [13].

More recently, the concept of Dutta multiplicity has been generalized to rings of arbitrary characteristic using localized Chern characters by Kurano [7]. While this definition agrees with the original one for rings of positive characteristic, the lack of a simple construction in terms of limits over the Frobenius map makes it more difficult to prove many of its properties. In this paper we show that the positivity result mentioned above can be generalized to homomorphic images of regular local rings of arbitrary characteristic containing a field.

The basic idea is to reduce to the case of positive characteristic using the "Metatheorem" of Hochster 6], which states that if a set of equations has a solution in any ring containing a field, then it has a solution in some ring of positive characteristic. To apply this theorem, we use an alternate description of Dutta multiplicity in terms of Adams operations. The theory of Adams operations can be considered to be a generalization of the Frobenius map to arbitrary characteristic, but it is defined on the Grothendieck group of complexes rather than induced by a map of rings, so the theory, like that of Dutta multiplicities, is somewhat more difficult than in the case of positive characteristic.

In the first section we give a precise statement of the theorem and introduce notation. In the next few sections we prove the necessary statements on Adams

Received by the editors April 10, 1998.

1991 Mathematics Subject Classification. Primary 13A35, 13D15; Secondary 14C17, 14 C35.

The first author would like to thank the University of Utah for its invitation during 1997-1998.

Both authors were supported in part through a grant from the National Science Foundation. 
operations, after which we prove the positivity result. In Section 5$]$ we prove a generalization of a result of Dutta on the positivity of intersection multiplicities.

\section{Notation And RESUlts}

In this section we state the main result on the positivity of Dutta multiplicity. We first recall the original definition of Dutta multiplicity in positive characteristic.

Let $A$ be a local ring, and let $\mathbb{F}$. be a bounded complex of $A$-modules. If $\mathbb{F}$. has homology of finite length, we define the Euler characteristic $\chi(\mathbb{F}$.) to be

$$
\chi(\mathbb{F} .)=\sum_{t}(-1)^{t} \ell\left(H_{t}(\mathbb{F} .)\right)
$$

where $H_{t}(\mathbb{F}$. $)$ is the $t$-th homology module of $\mathbb{F}$. and $\ell\left(H_{t}(\mathbb{F}\right.$. $\left.)\right)$ denotes its length.

Let $A$ be a complete local ring of positive characteristic $p$ with perfect residue field, and let $\mathbb{F}$. be a bounded complex of finitely generated free $A$-modules with homology of finite length. Let $d$ be the dimension of $A$. If we denote $\mathbb{F}{ }^{e}$ the tensor product of $\mathbb{F}$. with the $e$-th power of the Frobenius map, then the Dutta multiplicity is defined to be

$$
\chi_{\infty}(\mathbb{F} .)=\lim _{e \rightarrow \infty} \frac{\chi\left(\mathbb{F} .^{e}\right)}{p^{d e}} .
$$

Let $X$ be a scheme of finite type over a regular scheme. A bounded complex of locally free $\mathcal{O}_{X}$-modules of finite rank is called a perfect complex. For a perfect $\mathcal{O}_{X}$-complex $\mathbb{F}$., we define the support of $\mathbb{F}$. by

$$
\operatorname{Supp}(\mathbb{F} .)=\bigcup_{t} \operatorname{Supp}\left(H_{t}(\mathbb{F} .)\right) \text {. }
$$

The support of $\mathbb{F}$. is a closed set of $X$ consisting of those points at which $\mathbb{F}$. is not exact. Let $\mathbb{F}$. be a perfect $\mathcal{O}_{X}$-complex with support in $Y$. We denote the localized Chern character with respect to the perfect complex $\mathbb{F}$. by

$$
\operatorname{ch}_{Y}^{X}(\mathbb{F} .)=\bigoplus_{i \geq 0} \operatorname{ch}_{i}(\mathbb{F} .)
$$

If no confusion is possible, we denote it simply by $\operatorname{ch}(\mathbb{F}$.). We refer the reader to Fulton [3] or Roberts [14] for the definition and basic properties of localized Chern characters. We recall that localized Chern characters are defined as operators on the Chow group, and that if $\eta$ is a cycle of dimension $j$ in $\mathrm{A}_{j}(X)_{\mathbb{Q}}$, then $\operatorname{ch}_{i}(\mathbb{F}$. $)(\eta)$ is an element of $\mathrm{A}_{j-i}(Y)_{\mathbb{Q}}$.

Definition 1.1. Let $(A, m)$ be a homomorphic image of a regular local ring. Put $d=\operatorname{dim} A$. Let $\mathbb{F}$. be a perfect $A$-complex with support in $\{m\}$. Then, we define the Dutta multiplicity of a perfect $A$-complex $\mathbb{F}$. by letting

$$
\chi_{\infty}(\mathbb{F} .)=\operatorname{ch}_{d}(\mathbb{F} .) \cap[\operatorname{Spec} A] .
$$

By definition, $\chi_{\infty}(\mathbb{F}.) \in \mathrm{A}_{0}(\operatorname{Spec} A / m)_{\mathbb{Q}}=\mathbb{Q} \cdot[\operatorname{Spec} A / m]$. Hence, we may regard the Dutta multiplicity $\chi_{\infty}(\mathbb{F}$.) as a rational number.

Remark 1.2. Let $(A, m)$ be a complete equi-characteristic Noetherian local ring such that the residue class field is perfect of positive characteristic. Then the Dutta multiplicity $\chi_{\infty}(\mathbb{F}$.) as above coincides with the original one defined by Dutta [1] using the Frobenius endomorphism (Szpiro [16], Roberts [13], [14]). 
We also make one comment on the relation of Dutta multiplicity to the usual Euler characteristic. Let $X=\operatorname{Spec} A$, where $A$ has dimension $d$. Let $[\operatorname{Spec} A]$ be the element of the Chow group of $A$ in dimension $d$ defined by taking the sum of $\ell_{A_{\mathfrak{p}}}\left(A_{\mathfrak{p}}\right)[\operatorname{Spec} A / \mathfrak{p}]$, where the sum is taken over all prime ideals of $A$ with $\operatorname{dim} A / \mathfrak{p}=d$. By the local Riemann-Roch theorem (Fulton [3], Example 18.3.12), there is an element of $\mathrm{A}_{*}(X)_{\mathbb{Q}}$ denoted

$$
\tau_{A}(A)=\tau_{d}(A)+\tau_{d-1}(A)+\cdots+\tau_{0}(A),
$$

where $\tau_{i}(A) \in \mathrm{A}_{i}(X)_{\mathbb{Q}}$ for each $i, \tau_{d}(A)=[\operatorname{Spec} A]$, and

$$
\chi(\mathbb{F} .)=\sum_{i=0}^{d} \operatorname{ch}_{i}(\mathbb{F} .)\left(\tau_{i}(A)\right) .
$$

We refer to $\tau_{A}$ as the Riemann-Roch map. Comparing this expression for the Euler characteristic to Dutta multiplicity, we see that the Dutta multiplicity of $\mathbb{F}$. is equal to the term in this sum for which $i=d$. If $\tau_{i}(A)=0$ for $i<d$ (a situation which sometimes occurs; see the discussion at the end of Section 5), the Euler characteristic and the Dutta multiplicity of $\mathbb{F}$. are equal.

The main aim in the present paper is to prove the following theorem:

Theorem 1.3. Let $(A, m)$ be a homomorphic image of a regular local ring. Assume that $A$ contains a field. Put $d=\operatorname{dim} A$. Let

$$
\mathbb{F} .: 0 \rightarrow F_{d} \rightarrow \cdots \rightarrow F_{0} \rightarrow 0
$$

be a perfect $A$-complex with $\operatorname{Supp}(\mathbb{F})=.\{m\}$. (In particular, the support is not empty, and $\mathbb{F}$. is not exact.) Then we have $\chi_{\infty}(\mathbb{F}$. $)>0$.

The above theorem was proved by the second author 13 if $(A, m)$ is a complete equi-characteristic Noetherian local ring such that the residue class field is perfect of positive characteristic. It played an essential role in his proof of the New Intersection Theorem in the mixed characteristic case. By Theorem 1.3, we know that this proof of the New Intersection Theorem is valid for all Noetherian local rings.

It is open whether Theorem 1.3 is true or not without the assumption that $A$ contains a field. If $A$ has a test module in the sense of [10], then we do not need the assumption that $A$ has equal characteristic. We refer the reader to [10] for definition and basic properties of test modules.

We prove Theorem 1.3 in Section 4 .

Describing Theorem 1.3 in geometric terms, we obtain the following corollary.

Corollary 1.4. Let $X$ be a scheme of finite type over a regular scheme and $Y$ a closed subset of $X$. Let $d$ be a positive integer. Suppose that $\mathbb{F}$. is a perfect $\mathcal{O}_{X^{-}}$ complex with $\operatorname{Supp}(\mathbb{F}$. $)=Y$. Let $Z$ be a closed integral subscheme of $X$ and $W$ an irreducible component of $Z \cap Y$ such that $\operatorname{codim}_{Z} W=d$. Assume that $\mathbb{F}$. $\otimes_{\mathcal{O}_{X}} \mathcal{O}_{Z, W}$ is quasi-isomorphic to a perfect $\mathcal{O}_{Z, W}$-complex of length $d$. (A perfect complex $\mathbb{G}$. is said to be of length $d$ if $G_{i}=0$ for $i \notin[0, d], G_{0} \neq 0$ and $G_{d} \neq 0$.)

If the local ring $\mathcal{O}_{Z, W}$ contains a field, then the coefficient of $[W]$ in $\operatorname{ch}_{d}(\mathbb{F}$. $) \cap[Z]$ $\in \mathrm{A}_{*}(Y \cap Z)_{\mathbb{Q}}$ is positive.

Proof. This corollary is a translation of Theorem 1.3 into geometric language; we leave the details to the reader.

q.e.d.

In Section 5 we give an application of Theorem 1.3 to intersection multiplicities. 


\section{AdAMS OpERATIONS}

In this section we collect the facts about Adams operations which will be necessary for the proof of Theorem [1.3 We refer the reader to Gillet-Soulé [4] or Soulé 15. for details on the definition and basic properties of Adams operations.

For a closed subset $Y$ of $X$, we denote by $\mathrm{K}_{0}^{Y}(X)$ the Grothendieck group of perfect $\mathcal{O}_{X}$-complexes with supports in $Y$, i.e., $\mathrm{K}_{0}^{Y}(X)=Z / R$, where $Z$ is the free abelian group generated by the set of perfect $\mathcal{O}_{X}$-complexes whose supports are contained in $Y$ and $R$ is the subgroup of $Z$ generated by the following two types of relations:

- $[\mathbb{F}$.] - [G.]. if there exists a quasi-isomorphism from $\mathbb{F}$. to $\mathbb{G}$.;

- $[\mathbb{F}]+.[\mathbb{H}]-.[\mathbb{G}]$, if there is an exact sequence of complexes

$$
0 \rightarrow \mathbb{F} . \rightarrow \mathbb{G} . \rightarrow \mathbb{H} \text {. } \rightarrow 0 .
$$

Adams operations are defined on $\mathrm{K}_{0}^{Y}(X)$ using the $\lambda$-ring structure on $\mathrm{K}_{0}^{Y}(X)$, which is in turn defined by defining operators $\lambda^{r}$ on $\mathrm{K}_{0}^{Y}(X)$ using exterior powers. It has been shown by M. Hashimoto (unpublished) that if the underlying ring contains a field of characteristic zero, the $\lambda$-operations can be defined directly using exterior powers of complexes. In general, however, a more complicated definition using simplicial objects is required. We refer to Gillet-Soulé [4 for details of this construction. The properties of $\lambda^{r}$ we need are summarized in the following proposition.

Proposition 2.1. $\quad$ 1. If $\mathbb{F}$. is a perfect complex with $F_{t}=0$ for $t \neq 0$, then $\lambda^{r}\left(\mathbb{F}\right.$.) is the $r$-th exterior power of $F_{0}$ (in degree zero).

2. If $\mathbb{F}$. is a complex of the form

$$
\cdots \rightarrow 0 \rightarrow \mathcal{O}_{X}(-D) \rightarrow \mathcal{O}_{X} \rightarrow 0 \rightarrow \cdots,
$$

where $\mathcal{O}_{X}(-D)$ and $\mathcal{O}_{X}$ have degrees 1 and 0 respectively, then

$$
\left[\lambda^{r}(\mathbb{F} .)\right]=(-1)^{r-1}\left[\mathbb{F} . \otimes \mathcal{O}_{X}(-(r-1) D)\right]
$$

in $\mathrm{K}_{0}^{Y}(X)$.

3. Let $\mathbb{F}$. be a perfect complex of free modules over a local ring, and let the rank of $F_{t}$ be $r_{t}$ for each $t$. Then $\lambda^{r}(\mathbb{F}$.) can be represented by a complex $\mathbb{G}$., where the rank of $G_{i}$ for each $i$ depends only on the values of $r_{t}$, and where the boundary maps are defined by matrices with entries which are polynomials in the entries of the matrices of $\mathbb{F}$. with coefficients in $\mathbb{Z}$. These polynomials also depend only on the numbers $r_{t}$.

Proof. We refer to Gillet and Soulé [4] for verification of these facts. The second statement is a global version of their Lemma 4.12.

q.e.d.

For a closed subset $Y$ of $X$ and an integer $k \geq 1$, we next define a map $\psi^{k}$ : $\mathrm{K}_{0}^{Y}(X) \rightarrow \mathrm{K}_{0}^{Y}(X)$, which is called the $k$-th Adams operation. Adams operations are defined in terms of the $\lambda$-operations by the following formula, where $U$ denotes the operation induced by the tensor product of complexes.

$$
\psi^{k}-\psi^{k-1} \cup \lambda^{1}+\cdots+(-1)^{k-1} \psi^{1} \cup \lambda^{k-1}+(-1)^{k} k \lambda^{k}=0 .
$$

Adams operations are functorial with respect to pullback of perfect complexes and are multiplicative with respect to the tensor product. We note also the localized Chern characters are additive with respect to short exact sequences, multiplicative 
with respect to the tensor product, and functorial with respect to flat pullback and proper push-forward (see Fulton [3], Chapter 18).

\section{The relation between Adams operations AND LOCALIZED CHERN CHARACTERS}

In this section we prove the following theorem.

Theorem 3.1. Let $X$ be a scheme of finite type over a regular scheme. Let $Y$ be a closed subset of $X$ and let $\mathbb{F}$. be a perfect $\mathcal{O}_{X}$-complex with support in $Y$. Then, for any $k \geq 1$ and $i \geq 0$, we have

$$
\operatorname{ch}_{i}\left(\psi^{k}(\mathbb{F} .)\right)=k^{i} \operatorname{ch}_{i}(\mathbb{F} .)
$$

The proof of Theorem 3.1 uses the splitting principle for complexes and an explicit computation of Adams operations in the case of an elementary complex. We recall that an elementary complex is a complex of one of the two forms:

1. A locally free sheaf $\mathcal{L}$ of rank one in degree zero.

2. A complex of the form

$$
0 \rightarrow \mathcal{L} \otimes \mathcal{O}_{X}(-D) \rightarrow \mathcal{L} \rightarrow 0,
$$

where $D$ is an effective Cartier divisor and $\mathcal{L}$ is a locally free sheaf of rank one.

By the splitting principle for complexes (see Fulton [3], Example 18.3 .12 or Roberts [14], section 12.2), there exists a projective map from $Y$ to $X$ such that the pullback of $\mathbb{F}$. to $Y$ has a filtration with quotients which are elementary complexes with shifts of degrees. Thus, using the functorial properties of localized Chern characters and of Adams operations, together with their additivity, it suffices to prove the result in the case of an elementary complex (see either of the references cited above for the details of this procedure).

We first compute the Adams operations for elementary complexes.

Lemma 3.2. Let $X$ be a scheme.

(a) Let $\mathcal{L}$ be a line bundle (locally free sheaf of rank 1 ). Then, for any $k \geq 1$, we have $\psi^{k}(\mathcal{L})=\mathcal{L}^{\otimes k}$, where we regard $\mathcal{L}$ as a complex concentrated in degree 0 .

(b) Let $D$ be an effective Cartier divisor on $X$. For a positive integer n, we define a perfect complex $\mathbb{E}^{(n)}$ with support $D$ as

$$
E_{t}^{(n)}= \begin{cases}\mathcal{O}_{X} & \text { if } t=0 \\ \mathcal{O}_{X}(-n D) & \text { if } t=1, \\ 0 & \text { otherwise }\end{cases}
$$

where $\mathcal{O}_{X}(-n D) \rightarrow \mathcal{O}_{X}$ is the natural inclusion. We put $\mathbb{E} .=\mathbb{E}^{(1)}$. Then,

$$
\left[\mathbb{E}^{(n)}\right]=[\mathbb{E} .]+\left[\mathbb{E} . \otimes \mathcal{O}_{X}(-D)\right]+\cdots+\left[\mathbb{E} . \otimes \mathcal{O}_{X}(-(n-1) D)\right]
$$

in $\mathrm{K}_{0}^{D}(X)$.

(c) With notation as above, for any $k \geq 1, \psi^{k}(\mathbb{E}$. $)=\left[\mathbb{E}^{(k)}\right]$.

Proof. By the first part of Proposition 2.1 we have

$$
\lambda^{k}(\mathcal{L})= \begin{cases}\mathcal{L} & \text { if } k=1 \\ 0 & \text { if } k \geq 2\end{cases}
$$

Therefore, by equation (2) of Section 2, we obtain $\psi^{k}(\mathcal{L})=\mathcal{L}^{\otimes k}$ for any $k \geq 1$. 
To prove (b), we take the total complex of

$$
\begin{array}{ccc}
\mathcal{O}_{X}(-n D) & = & \mathcal{O}_{X}(-n D) \\
\downarrow & & \downarrow \\
\mathcal{O}_{X}(-(n-1) D) & \rightarrow & \mathcal{O}_{X} .
\end{array}
$$

Taking the filtration of this double complex by rows, and using that the top row is exact, we see that the total complex is quasi-isomorphic to $\mathbb{E}^{(n-1)}$. Taking filtration by columns, we conclude that it is also equal to

$$
\left[\mathbb{E}^{(n)}\right]-\left[\mathbb{E} . \otimes \mathcal{O}_{X}(-(n-1) D)\right]
$$

in $\mathrm{K}_{0}^{D}(X)$. Therefore, (b) follows by induction on $n$.

We now prove (c) by induction on $k$. By Proposition 2.1 we have

$$
\lambda^{k}(\mathbb{E} .)=(-1)^{k-1}\left[\mathbb{E} . \otimes \mathcal{O}_{X}(-(k-1) D)\right]
$$

for $k \geq 1$. In particular, we have $\psi^{1}(\mathbb{E}$. $)=\mathbb{E}$. $=\lambda^{1}(\mathbb{E}$.). Suppose $k \geq 2$. Assertion (c) follows easily from (b), equation (2) of Section 2 and the fact, proven in the same way as $(\mathrm{b})$, that $[\mathbb{E} . \otimes \mathbb{E}]=.[\mathbb{E}]-.\left[\mathbb{E} . \otimes \mathcal{O}_{X}(-D)\right]$.

q.e.d.

We now prove Theorem 3.1 for elementary complexes. We may assume that $X$ is an integral scheme, and we have only to prove that

$$
\operatorname{ch}_{i}\left(\psi^{k}(\mathbb{F} .)\right) \cap[X]=k^{i} \operatorname{ch}_{i}(\mathbb{F} .) \cap[X]
$$

for $k \geq 1$ and $i \geq 0$.

First, assume that $F_{0}$ is a line bundle and $F_{t}=0$ for $t \neq 0$. In this case the localized Chern character of $\mathbb{F}$. is the Chern character of $F_{0}$ as a sheaf, and (by Proposition 18.1 (a) in [3] or [14], section 11.4), we have

$$
\operatorname{ch}_{i}(\mathbb{F} .)=\operatorname{ch}_{i}\left(F_{0}\right)=\frac{1}{i !} c_{1}\left(F_{0}\right)^{i}
$$

where $c_{1}\left(F_{0}\right)$ is the first Chern class of the line bundle $F_{0}$. Therefore, by Lemma 3.2 (a), we have

$$
\operatorname{ch}_{i}\left(\psi^{k}(\mathbb{F} .)\right)=\operatorname{ch}_{i}\left(F_{0}^{\otimes k}\right)=\frac{1}{i !}\left\{k \cdot c_{1}\left(F_{0}\right)\right\}^{i}=k^{i} \operatorname{ch}_{i}(\mathbb{F} .) .
$$

Next, we suppose that $Y \neq X$ and that $\mathbb{F}$. is an elementary complex concentrated in degrees 0 and 1 . Then, there exist a line bundle $\mathcal{L}$ and an elementary complex $\mathbb{E}$. defined by an effective Cartier divisor $D$ as in Lemma $3.2(\mathrm{~b})$ such that $\mathbb{F} .=\mathbb{E} . \otimes \mathcal{L}$. Note that $Y$ contains $D$ by definition. By the multiplicativity of localized Chern characters (Example 18.1.5 in Fulton [3]), we have

$$
\operatorname{ch}(\mathbb{F} .)=\operatorname{ch}(\mathcal{L}) \cdot \operatorname{ch}(\mathbb{E} .) .
$$

On the other hand, by the multiplicative property of Adams operations (4.11, A2) in Gillet and Soulé [4]) and Lemma 3.2 (c), we have

$$
\operatorname{ch}\left(\psi^{k}(\mathbb{F} .)\right)=\operatorname{ch}\left(\psi^{k}(\mathcal{L}) \otimes \psi^{k}(\mathbb{E} .)\right)=\operatorname{ch}\left(\psi^{k}(\mathcal{L})\right) \cdot \operatorname{ch}\left(\mathbb{E}^{(k)}\right) .
$$

By Corollary 18.1.2 in Fulton [3] or Theorem 11.4.5 in Roberts [14], we have

$$
\operatorname{ch}(\mathbb{E} .)=1-e^{-D} .
$$

Therefore, we have

$$
\begin{aligned}
\operatorname{ch}(\mathbb{F} .) & =\operatorname{ch}(\mathcal{L}) \cdot \operatorname{ch}(\mathbb{E} .)=e^{c} \cdot\left(1-e^{-D}\right), \\
\operatorname{ch}\left(\psi^{k}(\mathbb{F} .)\right) & =\operatorname{ch}\left(\psi^{k}(\mathcal{L})\right) \cdot \operatorname{ch}\left(\mathbb{E}^{(k)}\right)=e^{k c} \cdot\left(1-e^{-k D}\right),
\end{aligned}
$$


where $c=c_{1}(\mathcal{L})$. Since $\operatorname{ch}_{i}(\mathbb{F}$. $)\left(\operatorname{resp} . \operatorname{ch}_{i}\left(\psi^{k}(\mathbb{F}\right.\right.$. $\left.\left.)\right)\right)$ is the homogeneous component of $\operatorname{ch}\left(\mathbb{E}\right.$.) $\left(\right.$ resp. $\operatorname{ch}\left(\psi^{k}(\mathbb{F}\right.$. $\left.\left.)\right)\right)$ in degree $i$ with respect to the total degree in variables $c$ and $D$, we obtain $\operatorname{ch}_{i}\left(\psi^{k}(\mathbb{F}\right.$. $\left.)\right)=k^{i} \operatorname{ch}_{i}(\mathbb{F}$.). This completes the proof of Theorem 3.1.

q.e.d.

\section{Proof of the MAIN THEOREM}

In this section we prove Theorem 1.3 ,

If $A$ is a complete equi-characteristic local ring, and if the residue class field of $A$ is perfect of characteristic $p>0$, then $\chi_{\infty}(\mathbb{F}$.) coincides with the original Dutta multiplicity (Remark 1.2). Using this fact, the theorem was proven in this case by the second author [13, [14:

Now let $A$ be an arbitrary homomorphic image of a regular local ring containing a field of positive characteristic. By a standard construction (see [5], section 0.6.8), there exists a flat map $f: A \rightarrow B$ of relative dimension 0 such that $B$ is also a homomorphic image of a regular local ring and such that the residue field of $B$ is perfect. We recall (see Fulton [3], Appendix B.2.5) that to say that $f$ has relative dimension 0 means that for all prime ideals $\mathfrak{p}$ of $A$, and for all primes ideals $\mathfrak{q}$ of $B$ minimal over $f(\mathfrak{p}) B$, we have $\operatorname{dim} A / \mathfrak{p}=\operatorname{dim} B / \mathfrak{q}$. We can then complete the local ring $B$; the map from $B$ to its completion $\hat{B}$ is flat of relative dimension zero since a homomorphic image of a regular local ring is formally equidimensional (or quasi-unmixed; see Matsumura [11], Section 31). Then, using the compatibility of localized Chern classes with flat pullback (see Fulton [3] or Roberts [14]; although Fulton assumes that maps are of finite type, the proof is valid also in the case we are considering), letting $m$ denote the maximal ideal of $A$, we have

$$
\ell_{\hat{B}}(\hat{B} / m \hat{B}) \cdot \chi_{\infty}(\mathbb{F} .)=\chi_{\infty}\left(\mathbb{F} \cdot \otimes_{A} \hat{B}\right) .
$$

Hence, since $\chi_{\infty}\left(\mathbb{F}\right.$. $\left.\otimes_{A} \hat{B}\right)>0$, we have $\chi_{\infty}(\mathbb{F})>0$.

We now present the main part of the proof, which is the reduction of the characteristic zero case to the case of positive characteristic. The main theorem we use, as mentioned in the introduction, is the Metatheorem of Hochster [6], which states that if a system of equations has a solution over a ring of characteristic zero satisfying certain properties, then it has a solution over a ring of positive characteristic. The precise statement of this lemma in the form we need is as follows (Kurano [8]). In this lemma, a solution of an ideal $\mathfrak{a}$ of a polynomial ring means a sequence of elements which gives zero when evaluated at any polynomial in $\mathfrak{a}$.

Lemma 4.1. Let $\mathfrak{a}$ be an ideal of the polynomial ring

$$
\mathbb{Z}\left[Y_{1}, \ldots, Y_{n}, X_{1}, \ldots, X_{d}, G_{1}, \ldots, G_{l}, W_{1}, \ldots, W_{k}\right]
$$

over $\mathbb{Z}$. Suppose that a regular local ring $R$ containing $\mathbb{Q}$ has a solution

$$
y_{1}, \ldots, y_{n}, x_{1}, \ldots, x_{d}, g_{1}, \ldots, g_{l}, w_{1}, \ldots, w_{k}
$$

of $\mathfrak{a}$ such that $y_{1}, \ldots, y_{n}$ forms a regular system of parameters for $R$ and $\overline{x_{1}}, \ldots, \overline{x_{d}}$ forms a system of parameters for $R /\left(g_{1}, \ldots, g_{l}\right)$, where $\overline{x_{i}}$ stands for the homomorphic image of $x_{i}$ for each $i$. Then there exists a regular local ring $R^{\prime}$ which satisfies the following two conditions; (1) $R^{\prime}$ is essentially of finite type over a field of positive characteristic, (2) $R^{\prime}$ has a solution

$$
y_{1}^{\prime}, \ldots, y_{n}^{\prime}, x_{1}^{\prime}, \ldots, x_{d}^{\prime}, g_{1}^{\prime}, \ldots, g_{l}^{\prime}, w_{1}^{\prime}, \ldots, w_{k}^{\prime}
$$


of $\mathfrak{a}$ such that $y_{1}^{\prime}, \ldots, y_{n}^{\prime}$ forms a regular system of parameters for $R^{\prime}$ and $\overline{x_{1}^{\prime}}, \ldots, \overline{x_{d}^{\prime}}$ forms a system of parameters for $R^{\prime} /\left(g_{1}^{\prime}, \ldots, g_{l}^{\prime}\right)$.

Thus what we need to prove Theorem 1.3 is to express the positivity of Dutta multiplicity in terms of equations of the type described in Lemma 4.1.

We assume that we have a counterexample to the positivity of Dutta multiplicity over a ring $A$ which is a homomorphic image of a regular local ring and which contains a field of characteristic zero. Let the dimension of $A$ be $d$. Then there exists a complex

$$
\mathbb{F} .: 0 \rightarrow F_{d} \rightarrow \cdots \rightarrow F_{0} \rightarrow 0
$$

such that the Dutta multiplicity is not positive. We can represent the maps of free modules by matrices, and the fact that $\mathbb{F}$. is a complex is expressed by the fact that the entries in these matrices form the solution to a set of polynomials. We wish to show that we can add more variables and new polynomials to the system so that every solution to the new system defines a complex $\mathbb{G}$. such that

$$
\ell\left(H_{t}(\mathbb{G} .)\right)=\ell\left(H_{t}(\mathbb{F} .)\right)
$$

for all $t$, so that in particular we have $\chi(\mathbb{G}$. $)=\chi(\mathbb{F}$.). To accomplish this, we use the following lemma (Lemma 3.11 of Kurano [8]).

Lemma 4.2. Let $R$ be a regular local ring of dimension $n$, and let $B$ denote the quotient $R /\left(g_{1}, \ldots, g_{l}\right)$, where $\left(g_{1}, \ldots, g_{l}\right)$ is a proper ideal of $R$. Let $a_{i j}$ and $b_{k m}$ be elements in $R$ such that

$$
B^{p \stackrel{\left(\overline{\left.a_{i j}\right)}\right.}{\longrightarrow}} B^{q \stackrel{\overline{\left(b_{k m}\right)}}{\rightarrow}} B^{r}
$$

is a complex of B-free modules and B-linear maps. Assume that the homology group $H$ of the above complex has finite length. Then, for a sufficiently large $s$, there exists an ideal $\mathfrak{b}$ of the polynomial ring over $\mathbb{Z}$ with indeterminates

$Y_{1}, \ldots, Y_{n}$

$G_{1}, \ldots, G_{l}$,

$\left\{A_{i j}, B_{k m} \mid i=1, \ldots, q ; j=1, \ldots, p ; k=1, \ldots, r ; l=1, \ldots, q\right\}$,

$W_{1}, \ldots, W_{s}$,

which satisfies the following two conditions:

1. There exist $y_{1}, \ldots, y_{n}, w_{1}, \ldots, w_{s} \in R$ such that $y_{1}, \ldots, y_{n}$ form a regular system of parameters of $R$ and $\underline{y}, \underline{g},\left\{a_{i j}, b_{k m} \mid i, j, k, m\right\}, \underline{w}$ is a solution of $\mathfrak{b}$.

2. If a regular local ring $E$ has a solution $\underline{y}^{\prime}, \underline{g}^{\prime},\left\{a_{i j}^{\prime}, b_{k m}^{\prime} \mid i, j, k, m\right\}, \underline{w}^{\prime}$ of $\mathfrak{b}$ such that $y_{1}^{\prime}, \ldots, y_{n}^{\prime}$ form a regular system of parameters of $E$, then the sequence

$$
\left(E /\left(g_{1}^{\prime}, \ldots, g_{l}^{\prime}\right)\right)^{p} \stackrel{\overline{\left(a_{i j}^{\prime}\right)}}{\longrightarrow}\left(E /\left(g_{1}^{\prime}, \ldots, g_{l}^{\prime}\right)\right)^{q} \stackrel{\overline{\left(b_{k m}^{\prime}\right)}}{\longrightarrow}\left(E /\left(g_{1}^{\prime}, \ldots, g_{l}^{\prime}\right)\right)^{r}
$$

is a complex whose homology has length equal to the length of $H$.

We refer to Kurano [8] for the proof of this lemma. Using the lemma, we can add the condition that the Euler characteristic has a specific value to the list of properties expressed by the existence of a solution to the polynomials. We next wish to do the same for Adams operations. This uses the next proposition, which states that we can express the Adams operations applied to a complex $\mathbb{F}$. in terms of a specific set of polynomials (with coefficients in $\mathbb{Z}$ ) in the entries of the matrices defining $\mathbb{F}$.. This result is a direct consequence of Proposition 2.1] which states that the $\lambda$-operations can be defined by a finite set of polynomials, combined with the inductive definition of Adams operations in equation (2) of Section 2 , 
Proposition 4.3. Let $e \geq 1$ be an integer, and let $r_{t}, t \in \mathbb{Z}$ be integers such that $r_{t}=0$ for all but finitely many $t$. Then there exist integers $g_{t}^{e}$ and $h_{t}^{e}$ indexed by $t \in \mathbb{Z}$, zero for all but finitely many $t$, satisfying the following condition:

For each $t$, there are polynomials $P_{i j t}^{e}\left(i=1, \ldots, g_{t-1}^{e} ; j=1, \ldots, g_{t}^{e}\right)$ and polynomials $Q_{i j t}^{e}\left(i=1, \ldots, h_{t-1}^{e} ; j=1, \ldots, h_{t}^{e}\right)$ such that for every complex $\mathbb{F}$. such that $F_{t}$ has rank $r_{t}$ for each $t, \psi^{e}(\mathbb{F}$.) can be represented in the Grothendieck group by $[\mathbb{G}]-.[\mathbb{H}$.$] where the ranks of G_{t}$ and $H_{t}$ are $g_{t}^{e}$ and $h_{t}^{e}$ respectively, and where the entries in the matrices of the maps $G_{t} \rightarrow G_{t-1}$ and $H_{t} \rightarrow H_{t-1}$ are given by the polynomials $P_{i j t}^{e}$ and $Q_{i j t}^{e}$ respectively. The polynomials $P_{i j t}^{e}$ and $Q_{i j t}^{e}$ are polynomials (with coefficients in $\mathbb{Z}$ ) in the entries of the matrices which define the maps of the complex $\mathbb{F}$..

We emphasize that these polynomials depend only on e and the ranks of the free modules $F_{t}$.

Proof. We prove this result by induction on $e$. If $e=1, \psi^{e}(\mathbb{F}$. $)=\mathbb{F}$. and we may let $g_{t}^{e}=r_{t}, h_{t}^{e}=0$, and the polynomials are defined by letting $P_{i j t}^{1}\left(\ldots, a_{k l s}, \ldots\right)=$ $a_{i j t}$, where $a_{k l s}$ denotes the $(k, l)$-entry of the matrix of the map $F_{s} \rightarrow F_{s-1}$.

Assume now that $e>1$. We remark first that if we can find integers representing the ranks of free modules and polynomials giving the entries of the matrices as above for complexes $\mathbb{E}$. and $\tilde{\mathbb{E}}$., then we can do the same for $\mathbb{E}$. $\oplus \tilde{\mathbb{E}}$. and $\mathbb{E}$. $\otimes \tilde{\mathbb{E}}$.. Furthermore, for each integer $i$, we may use Proposition 2.1 to find such a complex and polynomials depending only on $i$ and the $r_{t}$ such that $\lambda^{i}(\mathbb{F}$.) is represented by a complex which we denote $\Lambda^{i}(\mathbb{F}$.). By induction, we assume that we have integers and polynomials as above defining complexes $\mathbb{G}^{i}$. and $\mathbb{H}$. such that $\psi^{i}(\mathbb{F}$. $)=$ $\left[\mathbb{G}^{i}.\right]-\left[\mathbb{H}^{i}\right.$. $]$ for $i=1, \ldots, e-1$.

The inductive definition of Adams operations in equation (2) states that we have

$$
\psi^{e}(\mathbb{F} .)=\psi^{e-1}(\mathbb{F} .) \cup \lambda^{1}(\mathbb{F} .)-\cdots+(-1)^{e-2} \psi^{1}(\mathbb{F} .) \cup \lambda^{e-1}(\mathbb{F} .)+(-1)^{e-1} e \lambda^{e}(\mathbb{F} .) .
$$

Using this formula we may now write a representative for $\psi^{e}(\mathbb{F}$.) in the Grothendieck group in terms of complexes satisying the required properties in terms of the specific complexes $\Lambda^{i}\left(\mathbb{F}\right.$.) representing $\lambda^{i}\left(\mathbb{F}\right.$.) for all $i$ and $\mathbb{G}^{i}$. and $\mathbb{H}^{i}$. such that $\left[\mathbb{G}^{i}\right]-\left[\mathbb{H}^{i}\right]$ represents $\psi^{i}(\mathbb{F}$.) for $i=1, \ldots, e-1$. We write this out specifically in case $e$ is even (the odd case is similar). If $e$ is even, we have that $\psi^{e}(\mathbb{F}$.) can be represented by $\left[\mathbb{G}^{e}\right]-\left[\mathbb{H}^{e}\right]$, where

$$
\mathbb{G}^{e} .=\left(\mathbb{G}^{e-1} \otimes \Lambda^{1}(\mathbb{F} .)\right) \oplus\left(\mathbb{H}^{e-2} \otimes \Lambda^{2}(\mathbb{F} .)\right) \oplus \cdots \oplus\left(\mathbb{G}^{1} \otimes \Lambda^{e-1}(\mathbb{F} .)\right)
$$

and

$$
\mathbb{H}^{e} .=\left(\mathbb{H}^{e-1} \otimes \Lambda^{1}(\mathbb{F} .)\right) \oplus\left(\mathbb{G}^{e-2} \otimes \Lambda^{2}(\mathbb{F} .)\right) \oplus \cdots \oplus\left(\mathbb{H}^{1} . \otimes \Lambda^{e-1}(\mathbb{F} .)\right) \oplus\left(\Lambda^{e}(\mathbb{F} .)^{\oplus e}\right) .
$$

This completes the proof.

Combining these results, we conclude that it suffices to show that we can express the Dutta multiplicity in terms of a finite number of Euler characteristics $\chi\left(\psi^{e}(\mathbb{F}).\right)$ for a finite number of values of $e$. We do this in the following lemma.

Lemma 4.4. Let $(A, m)$ be a homomorphic image of a regular local ring and put $\operatorname{dim} A=d$. Let $\mathbb{F}$. be a perfect $A$-complex with support in $\{m\}$.

1. For any $k \geq 2$, we have

$$
\chi_{\infty}(\mathbb{F} .)=\lim _{e \rightarrow \infty} \frac{\chi\left(\psi^{e}(\mathbb{F} .)\right)}{e^{d}}=\lim _{e \rightarrow \infty} \frac{\chi\left(\left(\psi^{k}\right)^{e}(\mathbb{F} .)\right)}{k^{d e}} .
$$


2. There exist rational numbers $a_{1}, \ldots, a_{d+1}$ (depending only on $d$ ) such that

$$
\chi_{\infty}(\mathbb{F} .)=\sum_{e=1}^{d+1} a_{e} \cdot \chi\left(\psi^{e}(\mathbb{F} .)\right) .
$$

Proof. Let $\tau_{A}: K_{0}$ Spec $A_{\mathbb{Q}} \rightarrow \mathrm{A}_{*}$ Spec $A_{\mathbb{Q}}$ be the Riemann-Roch map for $A$. Put $\tau_{A}(A)=\tau_{d}+\tau_{d-1}+\cdots+\tau_{0}$, where $\tau_{i} \in \mathrm{A}_{i} \operatorname{Spec} A_{\mathbb{Q}}$ for each $i$. Note that $\tau_{d}=[\operatorname{Spec} A]$ is satisfied by the top term property (Theorem 18.3 (5) in Fulton [3]). Then, by Example 18.3.12 in 3] and Theorem [3.1, we have

$$
\begin{aligned}
\chi\left(\psi^{e}(\mathbb{F} .)\right) & =\operatorname{ch}\left(\psi^{e}(\mathbb{F} .)\right) \cap \tau_{A}(A) \\
& =\sum_{i=0}^{d} \operatorname{ch}_{i}\left(\psi^{e}(\mathbb{F} .)\right) \cap \tau_{i} \\
& =\sum_{i=0}^{d} e^{i} \operatorname{ch}_{i}(\mathbb{F} .) \cap \tau_{i} \\
\chi\left(\left(\psi^{k}\right)^{e}(\mathbb{F} .)\right) & =\operatorname{ch}\left(\left(\psi^{k}\right)^{e}(\mathbb{F} .)\right) \cap \tau_{A}(A) \\
& =\sum_{i=0}^{d} \operatorname{ch}_{i}\left(\left(\psi^{k}\right)^{e}(\mathbb{F} .)\right) \cap \tau_{i} \\
& =\sum_{i=0}^{d} k^{i e} \operatorname{ch}_{i}(\mathbb{F} .) \cap \tau_{i} .
\end{aligned}
$$

The first statement immediately follows from the equations above.

Put $\alpha_{e}=\chi\left(\psi^{e}(\mathbb{F}).\right)$ and $\beta_{i}=\operatorname{ch}_{i}(\mathbb{F}.) \cap \tau_{i}$. Then we have

$$
\left(\begin{array}{c}
\alpha_{1} \\
\alpha_{2} \\
\vdots \\
\alpha_{d+1}
\end{array}\right)=\left(\begin{array}{cccc}
1 & 1 & \cdots & 1 \\
1 & 2 & \cdots & 2^{d} \\
\vdots & \vdots & \ddots & \vdots \\
1 & d+1 & \cdots & (d+1)^{d}
\end{array}\right)\left(\begin{array}{c}
\beta_{0} \\
\beta_{1} \\
\vdots \\
\beta_{d}
\end{array}\right) .
$$

We denote by $L$ the $(d+1)$ by $(d+1)$ matrix in the equation as above. Obviously $L$ is invertible. Let $a_{1}, \ldots, a_{d+1}$ be the last row of $L^{-1}$. Then, we have

$$
\chi_{\infty}(\mathbb{F} .)=\beta_{d}=\sum_{e=1}^{d+1} a_{e} \cdot \chi\left(\psi^{e}(\mathbb{F} .)\right) .
$$

Now, we combine the above results to prove Theorem 1.3 in the case $A$ contains a field of characteristic 0 .

Let $(A, m)$ be a homomorphic image of regular local ring that contains a field of characteristic 0 . Put $d=\operatorname{dim} A$. Let

$$
\mathbb{F} .: 0 \rightarrow F_{d} \rightarrow \cdots \rightarrow F_{0} \rightarrow 0
$$

be a perfect $A$-complex with $\operatorname{Supp}(\mathbb{F}$. $)=\{m\}$.

Let $\mathbb{G}^{1}, \ldots, \mathbb{G}^{d+1}, \mathbb{H}^{1}, \ldots, \mathbb{H}^{d+1}$ be perfect $A$-complexes with supports in $\{m\}$ such that $\psi^{e}(\mathbb{F})=.\left[\mathbb{G}^{e}.\right]-\left[\mathbb{H}^{e}\right.$. $]$ as in the discussion preceding Lemma 4.4. Then, by 
Lemma4.1, Lemma4.2 and Proposition 4.3, we can find a $d$-dimensional Noetherian local ring $\left(B, m^{\prime}\right)$ and perfect $B$-complexes

$$
\begin{aligned}
& \mathbb{E} .: 0 \rightarrow E_{d} \rightarrow \cdots \rightarrow E_{0} \rightarrow 0, \\
& \mathbb{R}^{1}, \ldots, \mathbb{R}^{d+1}, \mathbb{S}^{1}, \ldots, \mathbb{S}^{d+1}
\end{aligned}
$$

that satisfy the following four conditions:

(i) $B$ is essentially of finite type over a field of positive characteristic.

(ii) The supports of perfect $B$-complexes $\mathbb{E} ., \mathbb{R}^{1}, \ldots, \mathbb{R}^{d+1}, \mathbb{S}^{1}, \ldots, \mathbb{S}^{d+1}$ are contained in $\left\{\mathrm{m}^{\prime}\right\}$.

(iii) $\psi^{e}(\mathbb{E}$. $)=\left[\mathbb{R}^{e}\right]-\left[\mathbb{S}^{e}\right]$ for $e=1, \ldots, d+1$.

(iv) We have equalities $\ell_{A}\left(H_{t}(\mathbb{F}\right.$. $\left.)\right)=\ell_{B}\left(H_{t}(\mathbb{E}).\right), \ell_{A}\left(H_{t}\left(\mathbb{G}^{e} \cdot\right)\right)=\ell_{B}\left(H_{t}\left(\mathbb{R}^{e}\right)\right)$, and $\ell_{A}\left(H_{t}(\mathbb{H} \cdot)\right)=\ell_{B}\left(H_{t}\left(\mathbb{S}^{e} \cdot\right)\right)$ for any $t$ and $e=1, \ldots, d+1$.

Then, by the conditions (iii) and (iv), we have

$$
\chi\left(\psi^{e}(\mathbb{F} .)\right)=\chi\left(\mathbb{G}^{e} .\right)-\chi\left(\mathbb{H}^{e} .\right)=\chi\left(\mathbb{R}^{e}\right)-\chi\left(\mathbb{S}^{e} \cdot\right)=\chi\left(\psi^{e}(\mathbb{E} .)\right)
$$

for $e=1, \ldots, d+1$. Hence, since $\mathbb{E}$. is defined over a ring of positive characteristic, Theorem 1.3 is true for $\mathbb{E}$., so we have

$$
\chi_{\infty}(\mathbb{F} .)=\chi_{\infty}(\mathbb{E} .)>0 .
$$

Thus the Dutta multiplicity of $\mathbb{F}$. is positive.

q.e.d.

Remark 4.5. Compare equation (11) in Section 1 with (3) in Lemma 4.4.

For a Noetherian local ring $A$ containing a field of characteristic $p>0$ and a perfect $A$-complex $\mathbb{F}$., $F^{e}(\mathbb{F}$.) denotes a perfect $A$-complex defined by matrices whose entries are the $p^{e}$-th powers of those of the complex $\mathbb{F}$..

Then, by a remark following Proposition 4.13 in Gillet and Soulé [4, we have $\left(\psi^{p}\right)^{e}(\mathbb{F})=.F^{e}(\mathbb{F}$.) for $e>0$.

Furthermore, if $A$ is an equi-characteristic complete local ring with perfect residue field of characteristic $p$, then we have $\chi\left(\mathbb{F} .{ }^{e}\right)=\chi\left(F^{e}(\mathbb{F}).\right)$. Therefore we obtain $\left.\chi\left(\left(\psi^{p}\right)^{e}(\mathbb{F}).\right)=\chi\left(\mathbb{F}^{e}\right)^{e}\right)$ in this case.

\section{AN APPLICATION TO INTERSECTION MULTIPLICITIES}

In this section we prove a generalization of a result of Dutta 2] on the positivity of intersection multiplicities.

Corollary 5.1. Let $(A, m)$ be a homomorphic image of a regular local ring with $d=\operatorname{dim} A$. Assume that $\tau_{A}(A) \in \mathrm{A}_{d} \operatorname{Spec} A_{\mathbb{Q}}$. Let $M$ and $N$ be finitely generated $A$-modules such that

- $\operatorname{pd}_{A} M<\infty, \operatorname{pd}_{A} N<\infty$,

- $0<\ell_{A}\left(M \otimes_{A} N\right)<\infty$,

- $\operatorname{dim} M+\operatorname{dim} N=\operatorname{dim} A$,

- $\operatorname{depth} M=\operatorname{dim} M$,

- for any prime ideal $Q$ in the support of $N$ with $\operatorname{dim}(A / Q)=\operatorname{dim}(N), A / Q$ contains a field,

where $\operatorname{pd}_{A} M\left(\right.$ resp. $\left.\operatorname{pd}_{A} N\right)$ denotes the projective dimension of $M($ resp. $N)$. Then

$$
\sum_{i}(-1)^{i} \ell_{A}\left(\operatorname{Tor}_{i}^{A}(M, N)\right)>0
$$


Proof. Put $X=\operatorname{Spec} A, Y=\operatorname{Supp} M$ and $Z=\operatorname{Supp} N$. Then, we have $Y \cap Z=$ Spec $A / m$ (as a set) and $\operatorname{dim} Y+\operatorname{dim} Z=d$ by our assumption. Put $s=\operatorname{dim} Y$ and $t=\operatorname{dim} Z$.

Let $\mathbb{F}$. and $\mathbb{G}$. be minimal $A$-free resolutions of $M$ and $N$ respectively. Then $\mathbb{F}$. and $\mathbb{G}$. are perfect $A$-complexes with supports $Y$ and $Z$. Then, by the local Riemann-Roch formula (Fulton [3], Example 18.3.12), we have

$$
\begin{aligned}
\sum_{i \geq 0}( & -1)^{i} \ell_{A}\left(\operatorname{Tor}_{i}^{A}(M, N)\right) \cdot[\operatorname{Spec} A / m] \\
& =\operatorname{ch}(\mathbb{F} \cdot \otimes \mathbb{G} \cdot) \cap \tau_{A}(A) \\
& =\operatorname{ch}_{d}(\mathbb{F} \cdot \otimes \mathbb{G} \cdot) \cap \tau_{A}(A) \\
& =\sum_{p+q=d} \operatorname{ch}_{p}(\mathbb{F} \cdot) \operatorname{ch}_{q}(\mathbb{G} .) \cap \tau_{A}(A),
\end{aligned}
$$

where the second equality uses the assumption that $\tau_{A}(A) \in \mathrm{A}_{d} X_{\mathbb{Q}}$. Let $p$ and $q$ be nonnegative integers such that

1. $p+q=d$,

2. $\operatorname{ch}_{p}(\mathbb{F}.) \operatorname{ch}_{q}(\mathbb{G}.) \cap \tau_{A}(A) \neq 0$,

Since $0 \neq \operatorname{ch}_{q}(\mathbb{G}$. $) \cap \tau_{A}(A) \in \mathrm{A}_{d-q} Z_{\mathbb{Q}}$ and $\operatorname{dim} Z=t$, we have $0 \leq d-q \leq t$. On the other hand, by the commutativity of localized Chern characters [12], we have

$$
\operatorname{ch}_{p}(\mathbb{F} .) \operatorname{ch}_{q}(\mathbb{G} .) \cap \tau_{A}(A)=\operatorname{ch}_{q}(\mathbb{G} .) \operatorname{ch}_{p}(\mathbb{F} .) \cap \tau_{A}(A) .
$$

Therefore, reversing the roles of $p$ and $q$, we have $\operatorname{ch}_{p}(\mathbb{F}$. $) \cap \tau_{A}(A) \neq 0$, so $0 \leq$ $d-p \leq s$. Since $d-q \leq t$ and $d-p \leq s$, we have $p=t$ and $q=s$. Hence, we get

$$
\sum_{i \geq 0}(-1)^{i} \ell_{A}\left(\operatorname{Tor}_{i}^{A}(M, N)\right) \cdot[\operatorname{Spec} A / m]=\operatorname{ch}_{t}(\mathbb{F} .) \operatorname{ch}_{s}(\mathbb{G} .) \cap \tau_{A}(A) .
$$

Let $I$ be the annihilator of $N$, that is, $I=\{x \in A \mid x N=0\}$. Then, by the local Riemann-Roch formula, we have

$$
\tau_{A / I}(N)=\operatorname{ch}(\mathbb{G} .) \cap \tau_{A}(A) \in \mathrm{A}_{*}(\operatorname{Spec} A / I)_{\mathbb{Q}} .
$$

Therefore $\operatorname{ch}_{s}(\mathbb{G}.) \cap \tau_{A}(A)$ coincides with the top term of $\tau_{A / I}(N)$, that is,

$$
\operatorname{ch}_{s}(\mathbb{G} .) \cap \tau_{A}(A)=\sum_{Q} \ell_{A_{Q}}\left(N_{Q}\right) \cdot[\operatorname{Spec} A / Q] \in \mathrm{A}_{t}(\operatorname{Spec} A / I)_{\mathbb{Q}}
$$

by Theorem $18.3(5)$ in Fulton [3], where the sum is taken over all $Q \in \operatorname{Supp}(N)$ with $\operatorname{dim}(A / Q)=\operatorname{dim}(N)$.

Thus, we have

$$
\begin{aligned}
\operatorname{ch}_{t}(\mathbb{F} .) \operatorname{ch}_{s}(\mathbb{G} .) \cap \tau_{A}(A) & =\sum_{Q} \ell_{A_{Q}}\left(N_{Q}\right) \cdot \operatorname{ch}_{t}(\mathbb{F} .) \cap[\operatorname{Spec} A / Q] \\
& =\sum_{Q} \ell_{A_{Q}}\left(N_{Q}\right) \cdot \operatorname{ch}_{t}(\mathbb{F} . \otimes A / Q) \cap[\operatorname{Spec} A / Q] .
\end{aligned}
$$

On the other hand, by the Auslander-Buchsbaum formula, we have

$$
\operatorname{pd}_{A} M=\operatorname{depth} A-\operatorname{depth} M \leq d-s=t,
$$

since $\operatorname{dim} M=\operatorname{depth} M$ by our assumption. Therefore the length of the complex 
$\mathbb{F}$. $\otimes A / Q$ is less than or equal to $t=\operatorname{dim} N=\operatorname{dim} A / Q$. Note that each homology module of $\mathbb{F} . \otimes A / Q$ has finite length and $\mathbb{F} . \otimes A / Q$ is not exact. (By the New Intersection Theorem [13, $A$ must be a Cohen-Macaulay ring.) Since $A / Q$ is equicharacteristic, we have $\operatorname{ch}_{t}(\mathbb{F} \cdot \otimes A / Q) \cap[\operatorname{Spec} A / Q]>0$ for each $Q$ as above by Theorem 1.3. Thus the intersection multiplicity of $M$ and $N$ is positive.

q.e.d.

Remark 5.2. We note several cases in which the assumption that

$$
\tau_{A}(A) \in \mathrm{A}_{d} \operatorname{Spec} A_{\mathbb{Q}}
$$

is satisfied. Assume that $A$ is a homomorphic image of a regular local ring such that $\operatorname{dim} A=d$.

1. If $A$ is a complete intersection, then $\tau_{A}(A) \in \mathrm{A}_{d} \operatorname{Spec} A_{\mathbb{Q}}$ by Corollary 18.1 .2 in Fulton [3]. On the other hand, there exists a Gorenstein ring $A$ such that $\tau_{A}(A) \notin \mathrm{A}_{d} \operatorname{Spec} A_{\mathbb{Q}}$ (see [9]).

2. Let $S$ be a $d$-dimensional regular local ring such that a finite group $G$ acts on $S$. Assume that $A=S^{G}$ is the invariant subring. Since $\mathrm{A}_{*} \operatorname{Spec} S_{\mathbb{Q}} \simeq$ $K_{0} \operatorname{Spec} S_{\mathbb{Q}} \simeq \mathbb{Q}$ by the singular Riemann-Roch theorem, we have

$$
\mathrm{A}_{i} \operatorname{Spec} S_{\mathbb{Q}}=\left\{\begin{array}{cc}
\mathbb{Q} & (i=d) \\
0 & (i \neq d) .
\end{array}\right.
$$

On the other hand, by Example 1.7.6 in Fulton [3], we have $\mathrm{A}_{*} \operatorname{Spec} A_{\mathbb{Q}}=$ $\left(\mathrm{A}_{*} \operatorname{Spec} S_{\mathbb{Q}}\right)^{G}$. Therefore, we have $\mathrm{A}_{*} \operatorname{Spec} A_{\mathbb{Q}}=\mathrm{A}_{d} \operatorname{Spec} A_{\mathbb{Q}}$. In particular, $\tau_{A}(A) \in \mathrm{A}_{d} \operatorname{Spec} A_{\mathbb{Q}}$ is satisfied.

\section{REFERENCES}

[1] S. P. DutTa, Frobenius and multiplicities, J. Alg. 85 (1983), 424-448. MR 85f:13022

[2] S. P. Dutta, A special case of positivity, Proc. Amer. Math. Soc. 103 (1988), 344-346. MR 89e:13028

[3] W. Fulton, Intersection Theory, Springer-Verlag, Berlin, New York, 1984. MR 85k:14004

[4] H. Gillet And C. Soulé, Intersection theory using Adams operations, Invent. Math. 90 (1987), 243-277. MR 89h:14005

[5] A. Grothendieck and J. A. Dieudonné, Eléments de Géométrie Algébrique, SpringerVerlag, 1971. MR 55:5621

[6] M. HochsteR, Topics in the homological theory of modules over local rings, CBMS Regional Conference Series in Math. 24. Amer. Math. Soc., Providence, RI, 1975. MR 51:8096

[7] K. Kurano, An approach to the characteristic free Dutta multiplicities, J. Math. Soc. Japan 45 (1993), 369-390. MR 94d:13026

[8] K. Kurano, On the vanishing and the positivity of intersection multiplicities over local rings with small non complete intersection loci, Nagoya J. Math. 136 (1994), 133-155. MR 95k:13029

[9] K. Kurano, A remark on the Riemann-Roch formula on affine schemes associated with Noetherian local rings, Tôhoku Math. J. 48 (1996), 121-138. [MR 97c:14006

[10] K. Kurano, Test modules to calculate Dutta multiplicities, in preparation.

[11] H. Matsumura, Commutative Rings, Cambridge University Press, 1985.

[12] P. Roberts, Local Chern characters and intersection multiplicities, Algebraic geometry, Bowdoin, Proc. Sympos. Math., 46, Amer. Math. Soc., Providence, RI, 1985, 389-400, 1987. MR 89a:14011

[13] P. Roberts, Intersection theorems, Commutative algebra, Math. Sci. Res. Inst. Publ., 15, Springer, New York, Berlin, 1989, 417-436. MR 90j:13024

[14] P. Roberts. Multiplicities and Chern classes in local algebra, Cambridge University Press (1998). CMP 99:13 
[15] C. Soulé, Lectures on Arakelov Geometry, Cambridge Studies in Advanced Math. 33, Cambridge University Press, 1992. MR 94e:14031

[16] L. SzPiro, Sur la théorie des complexes parfaits, Commutative algebra (Durham 1981), London Math. Soc. Lecture Note Ser. 72 (1982), 83-90. MR 84m:13015

Department of Mathematics, Tokyo Metropolitan University, Minami-Ohsawa 1-1, HACHIOJI, TOKYO 192-0397, JAPAN

E-mail address: kurano@comp.metro-u.ac.jp

Department of Mathematics, University of Utah, Salt Lake City, Utah 84112

E-mail address: roberts@math.utah.edu 